\title{
Características sociodemográficas, clínicas y farmacológicas de adultos mayores con fracturas, en tres centros de referencia, Colombia
}

Sociodemographic, clinical and pharmacological characteristics of older adults with fractures, in three reference centers, Colombia

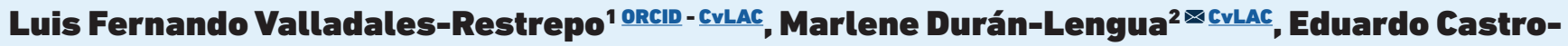 \\ Osorio $^{3 \text { ORCID }-~ C V L A C}$, Yessenia Correa- Sánchez ${ }^{4}$ ORCID , Jorge Enrique Machado-Alba ${ }^{3 \otimes ~ O R C I D}-$ CVLAC
}

Fecha correspondencia:

Recibido: julio 10 de 2019.

Revisado: septiembre 18 de 2019.

Aceptado: octubre 4 de 2019.

Forma de citar:

Valladales-Restrepo LF, Durán-

Lengua M, Castro-Osorio E,

Correa- Sánchez Y, Machado-

Alba JE. Características

sociodemográficas, clínicas

y farmacológicas de adultos

mayores con fracturas, en tres

centros de referencia, Colombia.

Rev CES Med 2020; 34(1): 14-26.

Open access

(C) Derecho de autor

Licencia creative commons

Ética de publicaciones

Revisión por pares

Gestión por Open Journal System

DOl: http://dx.doi.org/10.21615/

cesmedicina.34.1.2

Comparte

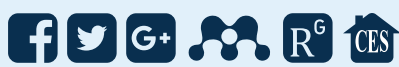

\section{Resumen}

Introducción: las caídas y fracturas óseas son causas importantes de morbilidad y mortalidad en ancianos. Su etiología es multicausal comprendiendo, entre otros, prescripciones inadecuadas y osteoporosis. El objetivo fue identificar las características clínicas y farmacológicas de un grupo de adultos mayores con fracturas. Materiales y métodos: estudio de corte transversal que analizó las prescripciones realizadas un mes antes de la fractura, durante la hospitalización y al egreso de 220 pacientes de 60 o más años atendidos en tres centros de referencia de Colombia. Se analizó la calidad de las prescripciones mediante los criterios STOPP. Resultados: la edad media fue de 75,3 $\pm 10,3$ años y $68,2 \%$ eran mujeres. La fractura de cadera fue la más frecuente $(37,7 \%)$ y se presentó una mortalidad intrahospitalaria del 2,3\%. La prescripción de suplementos de calcio/vitamina D y terapia antiabsortiva al egreso fue de $23,7 \%$ y $12,1 \%$, respectivamente. Las prescripciones potencialmente inapropiadas al egreso se identificaron en $11,6 \%$ de pacientes y tener cinco o más comorbilidades se asoció con mayor probabilidad de tenerlas (OR:9,44, IC95 \%: 2,16-41,20). Conclusiones: generalmente, el personal médico no formula medicamentos antiosteporóticos como prevención secundaria, ni mejoran la calidad de las prescripciones de los pacientes con fracturas una vez que son dados de alta, por lo que se hace necesario el desarrollo de estrategias farmacológicas para ayudar a prevenir nuevos episodios de caídas y fracturas.

Palabras clave: Fracturas óseas; Fractura de cadera; Osteoporosis; Anciano; Farmacoepidemiología.

\section{Abstract}

Introduction: Falls and bone fractures are important causes of morbidity and mortality in the elderly. Its etiology is multicausal, including inadequate prescriptions and osteoporosis. The aim was to identify the clinical and pharmacological characteristics of a group of older adults diagnosed with 
ISSN 0120-8705

e-ISSN 2215-9177

Sobre los autores:

1. Médico, MSc en

Farmacología. Grupo

de Investigación en

Farmacoepidemiología

y Farmacovigilancia,

Universidad Tecnológica de

Pereira-Audifarma S.A; Grupo

Biomedicina, Fundación

Universitaria Autónoma de

las Américas.

2. QF por Bacterióloga, PhD.

Universidad de Cartagena.

3. Especialista en Medicina

Interna, Especialista

en Geriatria. Grupo

de Investigación en

Farmacoepidemiología

y Farmacovigilancia,

Universidad Tecnológica de

Pereira-Audifarma S.A; SES

Hospital de Caldas.

4. Microbióloga. Grupo

de Investigación en

Farmacoepidemiología

y Farmacovigilancia.

Universidad Tecnológica de

Pereira-Audifarma.

5. Grupo de Investigación

en Farmacoepidemiología

y Farmacovigilancia.

Universidad Tecnológica de

Pereira-Audifarma S.A., fractures in Colombia. Methods: Cross-sectional study that analyzed the prescriptions made one month before the fracture, during hospitalization and discharge. Patients aged 60 years and over were selected, assisted in three reference centers in Colombia. The quality of the prescriptions was analyzed using the STOPP criteria. Results: 220 patients with fractures were included, with a mean age of $75.3 \pm 10.3$ years and $68.2 \%$ were women. Hip fracture was the most frequent (37.7 \%) and in-hospital mortality was $2.3 \%$. Prescription of calcium/vitamin D supplements and antiabsortive therapy at discharge was $23.7 \%$ and $12.1 \%$, respectively. Prescriptions potentially inappropriate to discharge were identified in $11.6 \%$ of patients and having five or more comorbidities was associated with greater probability of having them (OR:9.44, $95 \%$ Cl: 2.16-41.20). Conclusions: Medical staff generally do not formulate antiosteporotic medications as secondary prevention or improve the quality of prescriptions for fractured patients once they are hospitalized and discharged, so it is necessary to develop pharmacological strategies to help prevent new episodes of falls and fractures.

Keywords: Fractures bone; Hip fractures; Osteoporosis; Aged; Pharmacoepidemiology.

\section{Introducción}

El mundo está al borde de un hito demográfico. Se considera que el número de personas mayores de 65 años va a crecer de un estimado de 524 millones en 2010 a cerca de 1500 millones en 2050, con la mayor parte del incremento en los países en vías de desarrollo (1). En el censo colombiano realizado de 2018 se evidenció un incremento en la proporción de adultos mayores (9,2 \%, 4,186,000 habitantes) en comparación con lo encontrado en el año 2005 (6,3 \%, 2,612,500) (2). Esto evidencia que el país no es ajeno a los cambios demográficos y que en un futuro próximo, el aumento progresivo de la población geriátrica será una condición importante que requerirá una planificación económica, social y de salud pública (3).

La longevidad está asociada con mayor riesgo de tener enfermedades crónicas y con ello mayor uso de medicamentos (4). Su inadecuada prescripción en el paciente geriátrico es frecuente y se asocia a reacciones adversas que pueden ser prevenibles y que generan grandes costos para el sistema de salud (5).

Las caídas y fracturas son una de las principales causas de morbilidad y mortalidad en el adulto mayor. Uno de cada tres adultos mayores presentará al menos una caída cada año y se estima que 7 \% de ellas llevan a fractura (6). La Organización Mundial de la Salud (OMS) considera las caídas como un importante problema de salud pública, responsables de 646000 muertes al año en todo el mundo, 80 \% en los países con bajos ingresos (7).

La etiología de las caídas es multicausal e incluye comorbilidades (osteoartritis, osteoporosis, enfermedad de Parkinson, demencia, secuelas de accidente cerebrovascular, alteraciones visuales, hipotensión), uso de ciertos medicamentos, polifarmacia, prescripciones potencialmente inapropiadas, entre otras $(8,9)$.

El reconocimiento de las medicaciones inadecuadas y omitidas ha llevado al desarrollo de herramientas para medir la calidad de la formulación, entre ellos, los criterios STOPP/START (del inglés Screening Tool of Older Person's Prescriptions / Screening Tool to Alert doctors to Right Treatment). Algunas de las características que evalúa este instrumento involucran los medicamentos inapropiados, que son fármacos en los cuales el riesgo de su uso supera el potencial beneficio y, la prescripción 
La inadecuada prescripción de medicamentos en el paciente geriátrico es frecuente y se asocia a reacciones adversas que pueden ser prevenibles y que generan grandes costos para el sistema de salud. insuficiente de medicamentos que se refiere a la omisión de un fármaco cuando hay una indicación clara y no hay contraindicaciones $(10,11)$.

El conocimiento de las prescripciones potencialmente inapropiadas en la población colombiana no ha sido lo suficientemente caracterizado (12-14) y existe una falta de vigilancia más exhaustiva en el uso de los medicamentos y en el empleo adecuado de fármacos antiosteoporóticos, por lo que se planteó el objetivo de determinar las características sociodemográficas, clínicas y farmacológicas de un grupo de pacientes adultos mayores con diagnóstico de fracturas secundarias a caídas.

\section{Materiales y métodos}

Estudio de corte transversal de pacientes con diagnóstico de fracturas vertebrales y no vertebrales secundarias a caídas. Se analizaron las prescripciones realizadas un mes antes de la fractura, durante la hospitalización y al egreso, en el período comprendido entre el 1 de enero al 30 de junio de 2018. Se seleccionaron pacientes de 60 años o más, de cualquier sexo y atendidos en tres centros de referencia en Colombia, ubicados en Manizales y dos de Cartagena, que atienden población del régimen subsidiado y contributivo. Se excluyeron pacientes con fracturas de etiología infecciosa, oncológica, secundarias a accidente de tránsito y por arma de fuego.

La información fue recolectada mediante encuesta dirigida a cada paciente previa firma de consentimiento informado e historia clínica. Se diseñó una base de datos que permitió recoger estos grupos de variables: sociodemográficas, tipo de fractura, clínicas (manejo quirúrgico o no quirúrgico, estancia hospitalaria, comorbilidades y mortalidad intrahospitalaria), farmacológicas, criterios STOPP (se incluyeron 22 criterios distribuidos en varias secciones: indicación de la medicación ( $n=3)$, sistema nervioso central, uso de psicotrópicos $(n=14)$, fármacos que aumentan de forma predecible el riesgo de caídas en personas mayores $(n=4)$ y carga antimuscarínica anticolinérgica $(n=1)$ y comedicaciones.

Los datos se analizaron con el paquete estadístico SPSS (Statistical Package for the Social Sciences) Statistics, versión 24.0 para Windows (IBM, EE.UU). Se realizó un análisis descriptivo con frecuencias y proporciones para las variables cualitativas y medidas de tendencia central y de dispersión para las variables cuantitativas. Se determinó la comparación de variables cuantitativas mediante las pruebas t de Student o ANOVA y $X^{2}$ para las categóricas. Se realizaron modelos de regresión logística binaria usando como variable dependiente tener por lo menos un criterio STOPP en las prescripciones antes de la hospitalización y al egreso, y como covariables, las que se asociaron significativamente en los análisis bivariados. Se determinó como nivel de significación estadística una $p<0,05$.

El protocolo recibió el aval del Comité de Bioética de la Universidad Tecnológica de Pereira en la categoría de investigación sin riesgo. Se respetaron los principios éticos establecidos por la Declaración de Helsinki. No se consideraron datos personales de los pacientes. Se obtuvo autorización de cada uno de los centros de referencia implicados en la investigación.

\section{Resultados}

Fueron incluidos un total de 220 pacientes con fracturas. La edad media fue de $75,3 \pm 10,3$ años (rango: 60-103 años) y el 68,2 \% correspondió a mujeres. El 75,5\% no tenían conyugue y la escolaridad menor a cinco años se identificó en el 78,5\%, mientras que las 
La proporción de pacientes con fracturas no vertebrales fue de $93,6 \%$, siendo las fracturas de cadera, muñeca y húmero las más frecuentes. Y entre las fracturas vertebrales fueron las dorsolumbares. actividades del hogar figuraron como la ocupación más frecuente (44,5\%). El 4,1 \% no se encontraban afiliados al Sistema de Salud de Colombia (cuadro 1).

Cuadro 1. Características sociodemográficas

\begin{tabular}{|c|c|}
\hline Variables & $\%$ \\
\hline Edad, media en años \pm desv. estándar & $75,3 \pm 10,3$ \\
\hline Sexo (femenino/masculino) & $68,2 / 31,8$ \\
\hline \multicolumn{2}{|l|}{ Estado civil } \\
\hline Viudo & 40,5 \\
\hline Casado & 37,7 \\
\hline Soltero & 9,5 \\
\hline Separado & 6,4 \\
\hline Unión libre & 5,9 \\
\hline \multicolumn{2}{|l|}{ Escolaridad } \\
\hline Ninguna & 20,0 \\
\hline Primaria (completa/incompleta) & $30,0 / 34,5$ \\
\hline Secundaria (completa/incompleta) & $5,5 / 5,9$ \\
\hline Técnico (completa/incompleta) & $0,9 / 0$ \\
\hline Universitario (completa/incompleta) & $2,7 / 0,0$ \\
\hline Postgrado & 0,5 \\
\hline \multicolumn{2}{|l|}{ Ocupación } \\
\hline Ama de casa & 44,5 \\
\hline Cesante & 19,5 \\
\hline Pensionado & 17,3 \\
\hline Independiente & 8,2 \\
\hline Otros & 10,5 \\
\hline \multicolumn{2}{|c|}{ Régimen de afiliación en salud } \\
\hline Subsidiado & 50,9 \\
\hline Contributivo & 43,6 \\
\hline Especial & 1,4 \\
\hline No afiliado & 4,1 \\
\hline \multicolumn{2}{|c|}{ Institución prestadora de salud } \\
\hline Centro de referencia Cartagena 1 & 55,9 \\
\hline Centro de referencia Manizales & 31,8 \\
\hline Centro de referencia Cartagena 2 & 12,3 \\
\hline
\end{tabular}

Las comorbilidades más frecuentemente identificadas fueron hipertensión arterial (56,4 \%), osteoartrosis (15,5\%), diabetes mellitus (14,1\%), anemia (12,7\%), hipotiroidismo $(12,7 \%)$, demencia $(11,8 \%)$ y osteoporosis $(11,4 \%)$. La proporción de pacientes con fracturas no vertebrales fue de $93,6 \%$, siendo las fracturas de cadera, muñeca y húmero las más frecuentes. Y entre las fracturas vertebrales fueron las dorsolumbares (cuadro 2). 
Cuadro 2. Caracterización clínica y del manejo de las fracturas

\begin{tabular}{|c|c|}
\hline Variables & $\%$ \\
\hline \multicolumn{2}{|c|}{ Número de fracturas por paciente } \\
\hline Una & 91,4 \\
\hline Dos & 8,2 \\
\hline Tres & 0,5 \\
\hline \multicolumn{2}{|l|}{ Tipo de fractura } \\
\hline No vertebral & 93,6 \\
\hline - Cadera & 37,7 \\
\hline - Muñeca & 14,1 \\
\hline - Húmero* & 10,9 \\
\hline - Fémur* & 8,6 \\
\hline - Radio** & 6,8 \\
\hline - Tibia** & 6,4 \\
\hline - Hombro & 4,5 \\
\hline - Rotula & 2,7 \\
\hline - Tobillo & 2,7 \\
\hline - Cúbito** & 2,3 \\
\hline - Pelvis & 1,8 \\
\hline - Peroné** & 1,8 \\
\hline - Clavícula & 0,9 \\
\hline Vertebral & 7,3 \\
\hline - Lumbar & 5,0 \\
\hline - Dorsal & 0,9 \\
\hline - Cervical & 0,9 \\
\hline - Sacra & 0,5 \\
\hline \multicolumn{2}{|l|}{ Manejo } \\
\hline Quirúrgico & 88,6 \\
\hline No quirúrgico & 11,4 \\
\hline Estancia hospitalaria, días (RIC) & $8(4-14)$ \\
\hline Centro de referencia 1 Cartagena & $12(6-18)$ \\
\hline Centro de referencia Manizales & $5(6-18)$ \\
\hline Centro de referencia 2 Cartagena & $4(1-9)$ \\
\hline Mortalidad intrahospitalaria & 2,3 \\
\hline
\end{tabular}

El uso de antihipertensivos previo al ingreso hospitalario fue el grupo farmacológico más frecuente $(51,4 \%)$, mientras que a nivel intrahospitalario y al egreso fueron los analgésicos no opioides $(90,9 \%$ y 83,3\%). En las prescripciones del egreso, la terapia antiabsortiva se encontró en $12,1 \%$ y los suplementos de calcio/vitamina D fueron $23,7 \%$ (cuadro 3 ).

La formulación de terapias antiabsortivas y de suplementos de calcio/vitamina D se identificó principalmente en mujeres $(73,1 \%$, y $74,5 \%$, respectivamente). Entre los 83 pacientes con fractura de cadera, $20,5 \%$ recibió tratamiento antiabsortivo, al 
igual que calcio/vitamina $\mathrm{D}$; mientras que aquellos con fracturas no vertebrales el $23,3 \%$ recibió suplementos de calcio/vitamina D y 4,7 \% terapia antiabsortiva.

Cuadro 3. Principales grupos farmacológicos prescritos antes, durante y después de la atención hospitalaria (\%)

\begin{tabular}{lclclc}
\hline Grupo farmacológico & Antes $\mathbf{( n = 2 2 0 )}$ & Grupo farmacológico & Durante (n=220) & Grupo farmacológico & Después (n=215) \\
\hline Antihipertensivos & 51,4 & $\begin{array}{l}\text { Analgésicos no } \\
\text { opioides }\end{array}$ & 90,9 & $\begin{array}{l}\text { Analgésicos no } \\
\text { opioides }\end{array}$ & 83,3 \\
\hline $\begin{array}{l}\text { Analgésicos no } \\
\text { opioides }\end{array}$ & 25,0 & Antiulcerosos & 75,0 & Analgésicos opioides & 63,3 \\
\hline $\begin{array}{l}\text { Hipolipemiantes } \\
\text { Antiagregantes }\end{array}$ & 21,4 & Analgésicos opioides & 71,4 & Antiulcerosos & 57,7 \\
plaquetarios & 16,4 & Anticoagulantes & 68,2 & Antihipertensivos & 51,2 \\
\hline Antiulcerosos & 13,2 & Antihipertensivos & 45,0 & Anticoagulantes & 46,5 \\
\hline $\begin{array}{l}\text { Calcio/Vitamina D } \\
\text { Diuréticos }\end{array}$ & 12,7 & Antibióticos & 20,5 & Calcio/Vitamina D & 23,7 \\
\hline $\begin{array}{l}\text { Suplementos } \\
\text { vitamínicos }\end{array}$ & 11,8 & Hipolipemiantes & 15,5 & Antibióticos & 21,9 \\
\hline $\begin{array}{l}\text { Levotiroxina } \\
\text { Antidiabéticos orales }\end{array}$ & 11,8 & Diuréticos & 12,3 & Hipolipemiantes & 20,5 \\
\hline
\end{tabular}

Las prescripciones potencialmente inapropiadas relacionadas con el sistema nervioso central y con el riesgo de caídas se encontraron más frecuentemente durante la hospitalización.
De acuerdo con los criterios STOPP las prescripciones potencialmente inapropiadas relacionadas con el sistema nervioso central y con el riesgo de caídas se encontraron más frecuentemente durante la hospitalización (14,1\%), sin presentarse mayores diferencias entre el ingreso y el egreso (13,2 \% vs 11,6 \%) (cuadro 4).

Cuadro 4. Caracterización de las principales prescripciones potencialmente inapropiadas según los criterios STOPP (\%)

\begin{tabular}{lccc}
\hline Criterios STOPP & Antes $(\boldsymbol{n = 2 2 0})$ & Durante $(\boldsymbol{n = 2 2 0})$ & Después $(\boldsymbol{n = 2 1 5 )}$ \\
\hline $\begin{array}{l}\text { Anticolinérgicos en pacientes con delirium } \\
\text { o demencia }\end{array}$ & 7,3 & 7,3 & 6,5 \\
\hline Uso de neurolépticos & 7,3 & 6,8 & 7,0 \\
\hline Uso de benzodiazepinas & 3,2 & 3,6 & 3,7 \\
\hline $\begin{array}{l}\text { Cualquier prescripción concomitante de } \\
\text { dos fármacos de la misma clase }\end{array}$ & 2,7 & 3,6 & 1,9 \\
Antihistamínicos de primera generación & 1,8 & 1,4 & 0,5 \\
\hline Otras $(n=5)$ & 5,9 & 4,5 & 4,7
\end{tabular}

Mediante regresión logística se identificó que tener cinco o más comorbilidades aumentaba la probabilidad de que el paciente tuviera por lo menos una prescripción potencialmente inapropiada tanto al ingreso como al egreso (cuadro 5). 
Estos hallazgos pueden ser de utilidad ya que permitirán ilustrar los medicamentos de riesgo que pueden asociarse a desenlaces desafortunados y aumentar la morbilidad.
Cuadro 5. Análisis multivariado de las variables asociadas con la presencia de criterios STOPP

\begin{tabular}{lccc}
\hline & Sig. & OR & IC 95 \% \\
\hline Criterios STOPP al ingreso & & & \\
\hline Edad 60-74 años & 0,32 & Ref & Ref \\
\hline Edad 75-89 años & 0,42 & 1,52 & $0,55-4,23$ \\
\hline Edad $\geq 90$ años & 0,14 & 3,38 & $0,67-17,02$ \\
\hline Mujer & 0,80 & 0,88 & $0,34-2,32$ \\
\hline Régimen contributivo & 0,99 & 0,99 & $0,07-14,39$ \\
\hline Régimen subsidiado & 0,82 & 1,34 & $0,11-16,47$ \\
\hline Centro de referencia 1 & 0,93 & 0,88 & $0,05-14,61$ \\
\hline Centro de referencia 3 & 0,38 & 2,19 & $0,38-12,50$ \\
\hline 1-2 enfermedades & 0,68 & 0,76 & $0,20-2,85$ \\
\hline$\geq 5$ enfermedades & 0,00 & 6,40 & $1,83-22,39$ \\
\hline Diabetes mellitus & 0,55 & 1,41 & $0,47-4,23$
\end{tabular}

\section{Criterios STOPP al egreso}

\begin{tabular}{lccc}
\hline Edad 60-74 años & 0,24 & Ref & Ref \\
\hline Edad 75-89 años & 0,11 & 2,61 & $0,80-8,51$ \\
\hline Edad $\geq 90$ años & 0,25 & 3,25 & $0,44-24,13$ \\
\hline Mujer & 0,71 & 0,82 & $0,28-2,38$ \\
\hline Régimen contributivo & 0,82 & 0,71 & $0,04-12,59$ \\
\hline Régimen subsidiado & 0,92 & 1,16 & $0,07-18,57$ \\
\hline Centro de referencia 1 & 0,85 & 1,38 & $0,05-41,38$ \\
\hline Centro de referencia 3 & 0,35 & 2,97 & $0,30-29,16$ \\
\hline 1-2enfermedades & 0,39 & 0,47 & $0,08-2,62$ \\
\hline$\geq 5$ enfermedades & 0,00 & 9,45 & $2,17-41,20$ \\
\hline Diabetes mellitus & 0,78 & 1,19 & $0,35-4,02$ \\
\hline
\end{tabular}

STOPP: Herramienta de detección de prescripciones potencialmente inapropiadas de personas mayores, Sig: Significancia estadística, OR: Odss Ratio, IC: Intervalo de confianza

\section{Discusión}

Este estudio permitió documentar las características sociodemográficas y farmacológicas en los pacientes durante el mes previo a la fractura, en la hospitalización y al egreso, evaluando las prescripciones potencialmente inapropiadas mediante los criterios STOPP e identificando los fármacos antiabsortivos empleados como prevención secundaria posterior a una fractura. Estos hallazgos pueden ser de utilidad ya que permitirán ilustrar los medicamentos de riesgo que pueden asociarse a desenlaces desafortunados y aumentar la morbilidad.

La edad media de los pacientes con fracturas fue similar a lo reportado en otras publicaciones $(15,16)$. De igual manera, el predominio en mujeres también fue hallado en otros trabajos $(9,17,18)$. La fractura de cadera fue la más frecuente, con datos similares a lo descrito en Suecia (36 \%) (19), e inferiores a lo encontrado en España $(77,5 \%)(16)$, pero superiores a lo hallado en Norteamérica $(12,7-16,3 \%)(18,20)$. La 
Hay diversas comorbilidades asociadas con la probabilidad de aumentar el riesgo de caídas, en donde se destaca la hipertensión arterial, demencia, trastornos de la marcha o alteraciones de los sentidos. fractura de cadera se asocia a gran morbimortalidad, discapacidad, deterioro de la calidad de vida e incremento en los costos, tanto al sistema de salud como al paciente y a su entorno (21-23).

En otros estudios predomina la fractura de tercio distal de antebrazo (37,9-42,9\%) $(17,18)$, la cual es de gran importancia epidemiológica, debido a que los pacientes con antecedente de fractura de antebrazo distal o fractura vertebral tienen mayor riesgo de presentar una fractura de cadera que el resto de la población (24). Se desconocen por el momento publicaciones sobre el comportamiento epidemiológico de las fracturas a nivel local.

Hay diversas comorbilidades asociadas con la probabilidad de aumentar el riesgo de caídas, en donde se destaca la hipertensión arterial, demencia, trastornos de la marcha o alteraciones de los sentidos, entre otros $(8,9)$. La enfermedad más frecuentemente encontrada fue la hipertensión arterial, acorde a lo descrito en otras investigaciones $(9,25)$.

Un poco más de la mitad de los pacientes con fracturas se encontraba en tratamiento con antihipertensivos, lo que podría aumentar el riesgo de hipotensión como reacción adversa y asociarse con caídas $(26,27)$; sin embargo en el presente estudio no se obtuvieron las cifras tensionales al ingreso. De otro lado, la diabetes mellitus también puede asociarse con riesgo de caídas debido a complicaciones agudas o crónicas, y al riesgo de hipoglicemias que se puede presentar con los medicamentos empleados (28), sin embargo no se obtuvieron estudios paraclínicos para corroborar lo anterior.

La osteoporosis es una de las entidades más relacionadas con el riesgo de presentar fracturas; sin embargo, se ha evidenciado un marcado subdiagnóstico y por ende un inadecuado e inoportuno tratamiento (21-23). Su diagnóstico se realizó alrededor de una décima parte de los pacientes; sin embargo, es similar a lo encontrado en Malasia (9,4 \%) (15) y España (11,3\%) (29).

Se esperaría que la proporción en su diagnóstico fuera mayor en esta investigación debido al predominio femenino y a la alta edad media de la población. Es de resaltar, que si bien la osteoporosis es más frecuente en las mujeres postmenopáusicas, usualmente el diagnóstico y manejo en hombres pasa desapercibido $(7,22,23)$ como se evidenció en este reporte.

El tratamiento antiosteoporótico ha demostrado una reducción del 30-70 \% en el riesgo de fracturas vertebrales, de 30-50 \% para fracturas de cadera y de $25 \%$ en otras fracturas no vertebrales (30,31). El uso de calcio con o sin suplementos de vitamina $\mathrm{D}$ se asocia con una reducción del 12 \% del riesgo de fracturas de todo tipo (32). En este estudio, el 12,1\% de los pacientes recibieron terapia antiosteoporótica como prevención secundaria después de una fractura, siendo inferior a lo hallado en otros países $(23,9-38,5 \%)(33,34)$. El subtratamiento podría elevar el riesgo de nuevas caídas y de fracturas, impactando negativamente en la salud pública, puesto que entre un 15-25\% de ellos presentará una segunda fractura en los próximos 10 años (21).

La evidencia actual sugiere que la estrategia más efectiva para reducir la frecuencia de caídas en los adultos mayores es interviniendo múltiples factores de riesgo, en asuntos como el ejercicio, terapia física, instrucciones de comportamiento, cirugía 
Enero - abril de 2020 - Pág 22

Escasamente los médicos hacen ajustes terapéuticos acordes con el riesgo, interacciones y comorbilidades de cada paciente, por lo que se hace necesario establecer estrategias de prescripción para mejorar la calidad de las formulaciones. de cataratas, evaluación y modificación ambiental, reducción y ajustes de fármacos y mejorando la calidad de las prescripciones (35-37).

Se identificaron las prescripciones potencialmente inapropiadas mediante los criterios STOPP y se encontró que el $13,2 \%$ de los pacientes al ingreso presentaban por lo menos una prescripción asociada con el riesgo de caídas, lo cual fue inferior a lo reportado en otros estudios, aunque en estos se incluyeron todos los criterios (38-40). En Colombia, en un grupo de adultos mayores se encontraron en el 6,9 \% de los pacientes (12).

Este trabajo evidenció que las prescripciones potencialmente inapropiadas aumentaron durante la hospitalización y persistieron en una décima parte de los pacientes al egreso, denotando que, escasamente los médicos hacen ajustes terapéuticos acordes con el riesgo, interacciones y comorbilidades de cada paciente, por lo que se hace necesario establecer estrategias de prescripción para mejorar la calidad de las formulaciones. Sin embargo, esta conducta no está exenta a problemas como efectos rebote, cambios farmacocinéticos/farmacodinámicos de los medicamentos y a preocupaciones que se puedan generar en el paciente y/o familiares por suspender algún fármaco (41).

Entre las prescripciones potencialmente inapropiadas, el uso de neurolépticos fueron los más frecuentes siendo superior a lo encontrado en otros estudios (2,2-5,5\%) $(38,40)$, seguido de la prescripción de benzodiazepinas; lo que fue inferior a lo descrito en otras investigaciones en donde se emplearon entre el 6,5 \%-27,1 \% (38-40). El efecto sedante y anticolinérgico de muchos de estos medicamentos contribuye a caídas aumentando la probabilidad de fracturas, lo que fue evidenciado en pacientes colombianos quienes tenían como prescripciones benzodiazepinas u opioides (42) y medicamentos anticolinérgicos (43), así como en literatura internacional (9).

No obstante, no todas las prescripciones potencialmente inapropiadas se pueden evitar, por lo tanto, los médicos deben involucrar a los pacientes en la toma de decisiones e individualizar las formulaciones en función a las condiciones médicas, sociales, de calidad de vida y pronóstico, sopesando riesgo/beneficio.

Se reconocen algunas limitaciones en la interpretación de ciertos resultados, puesto que se captaron pacientes de solo tres hospitales, no se identificaron variables paraclínicas ni clínicas (por ejemplo signos vitales) al momento del ingreso; además el patrón y frecuencia de prescripción de los diferentes medicamentos puede cambiar considerablemente entre las diferentes ciudades de un mismo país, por lo que se hace necesario realizar un estudio multicéntrico para confirmar los hallazgos encontrados en este reporte. Existe el riesgo de que no se identificaran todos los medicamentos que el paciente recibía previamente a la fractura. Sin embargo, reúne una muestra de pacientes de los diferentes regímenes de afiliación al Sistema de Salud del país.

Finalmente, podemos concluir que el personal médico generalmente no formula medicamentos antiosteporóticos como prevención secundaria, ni mejoran la calidad de las prescripciones de los pacientes con fracturas una vez que son hospitalizados y dados de alta, por lo que se hace necesario el desarrollo de estrategias farmacológicas para ayudar a prevenir nuevos episodios de caídas y fracturas. 
Agradecimientos: A Victor Ávila Lagares por su colaboración en la recolección de la información.

Declaración de intereses. Los autores declaran no tener conflicto de interés.

Fuentes de financiación: el presente trabajo no recibió financiación.

\section{Bibliografía}

1. Rodrigues MC, Oliveira Cd. Drug-drug interactions and adverse drug reactions in polypharmacy among older adults: an integrative review. Rev Lat Am Enfermagem. 2016;24:e2800.

2. Departamento Administrativo Nacional de Estadística de Colombia (DANE). Censo general 2005 [internet]. [Consultado: 25 de Diciembre 2017]. Disponible en: http://www.dane.gov.co/files/censoslibroCenso2005nacional.pdf.

3. Gonzáles I, Becerra M, Gónzales J, Campos A, Barbosa J, Alvarado J. Fracturas de cadera: satisfacción posquirúrgica al año en adultos mayores atendidos en Méderi-Hospital Universitario Mayor, Bogotá, D.C. Rev Cienc Salud. 2015;14(3):409-22.

El personal médico generalmente no formula medicamentos antiosteporóticos como prevención secundaria, ni mejoran la calidad de las prescripciones de los pacientes con fracturas una vez que son hospitalizados y dados de alta.

4. Davies EA, O'Mahony MS. Adverse drug reactions in special populations - the elderly. Br J Clin Pharmacol. 2015;80(4):796-807.

5. Kim J, Parish AL. Polypharmacy and Medication Management in Older Adults. Nurs Clin North Am. 2017;52(3):457-468.

6. Court-Brown CM, McQueen MM. Global Forum: Fractures in the Elderly. J Bone Joint Surg Am. 2016;98(9):e36.

7. Lambert JK, Zaidi M, Mechanick JI. Male osteoporosis: epidemiology and thepathogenesis of aging bones. Curr Osteoporos Rep. 2011:9(4):229-36.

8. Berry SD, Miller R. Falls: Epidemiology, Pathophysiology, and Relationship to Fracture. Current osteoporosis reports. 2008;6(4):149-54.

9. Park H, Satoh H, Miki A, Maki H, Asai K, Shiraishi A, Urushihara H, Sawada Y. Medications and fall risk: a case-control study in nursing home residents in Japan. Aging Clin Exp Res. 2019. doi: 10.1007/s40520-019-01265-9. [Epub ahead of print].

10. Verdoorn S, Kwint HF, Faber A, Gussekloo J, Bouvy ML. Majority of drug-related problems identified during medication review are not associated with STOPP/ START criteria. Eur J Clin Pharmacol. 2015;71(10):1255-62.

11. Storms H, Marquet K, Aertgeerts B, Claes N. Prevalence of inappropriate medication use in residential long-term care facilities for the elderly: A systematic review. Eur J Gen Pract. 2017;23(1):69-77.

12. Cano-Guitierrez C, Samper-Ternent R, Cabrera J, Rosselli D. Medication use among older adults in Bogota, Colombia. Rev Peru Med Exp Salud Publica. 2016; 33(3):419-424. 
13. Machado-Duque M, Castro-Rodriguez A, Medina-Morales D, Machado-Alba J. Identification of Potentially Inappropriate Cardiovascular Prescriptions in the Elderly Using Beers Criteria in Colombia. Value in Health. 2018;21:S96.

14. Valladales-Restrepo LF, Duran-Lengua M, Machado-Alba JE. Potentially inappropriate prescriptions of anticholinergics drugs in Alzheimer's disease patients. Geriatr Gerontol Int. 2019;19(9):913-917.

15. Zia A, Kamaruzzaman SB, Tan MP. The consumption of two or more fall risk-increasing drugs rather than polypharmacy is associated with falls. Geriatr Gerontol Int. 2017;17(3):463-470.

16. Aguilar Del Rey FJ, Pérez González O. Epidemiology of osteoporotic fractures in Andalusia, Spain, from 2000-2010. Med Clin (Barc). 2018;150(8):297-302.

17. Liu SK, Munson JC, Bell JE, Zaha RL, Mecchella JN, Tosteson AN, Morden NE. Quality of osteoporosis care of older Medicare recipients with fragility fractures: 2006 to 2010. J Am Geriatr Soc. 2013;61(11):1855-62.

18. Roux S, Cabana F, Carrier N, Beaulieu M, April PM, Beaulieu MC, Boire G. The World Health Organization Fracture Risk Assessment Tool (FRAX) underestimates incident and recurrent fractures in consecutive patients with fragility fractures. $J$ Clin Endocrinol Metab. 2014;99(7):2400-8.

19. Hernlund E, Svedbom A, Ivergård M, Compston J, Cooper C, Stenmark J, et al. Osteoporosis in the European Union: medical management, epidemiology and economic burden. A report prepared in collaboration with the International Osteoporosis Foundation (IOF) and the European Federation of Pharmaceutical Industry Associations (EFPIA). Arch Osteoporos. 2013;8:136.

20. Tran T, Bliuc D, van Geel T, Adachi JD, Berger C, van den Bergh J, et al. Population-Wide Impact of Non-Hip Non-Vertebral Fractures on Mortality. J Bone Miner Res. 2017;32(9):1802-1810.

21. Desai RJ, Mahesri M, Abdia Y, Barberio J, Tong A, Zhang D, et al. Association of Osteoporosis Medication Use After Hip Fracture With Prevention of Subsequent Nonvertebral Fractures: An Instrumental Variable Analysis. JAMA Netw Open. 2018;1(3):e180826.

22. Dovjak P. [Diagnostics and treatment of osteoporosis in patients over 65 years old: Current status and future perspectives]. Z Gerontol Geriatr. 2019;52(5):421-427.

23. Narla RR, Hirano LA, Lo SHY, Anawalt BD, Phelan EA, Matsumoto AM. Suboptimal osteoporosis evaluation and treatment in older men with and without additional high-risk factors for fractures. J Investig Med. 2019;67(4):743-749.

24. Haentjens P, Autier P, Collins J, Velkeniers B, Vanderschueren D, Boonen S. Colles fracture, spine fracture, and subsequent risk of hip fracture in men and women. A meta-analysis. J Bone Joint Surg Am. 2003;85(10):1936-43. 
25. Perng HJ, Chiu YL, Chung CH, Kao S, Chien WC. Fall and risk factors for veterans and non-veterans inpatients over the age of 65 years: 14 years of long-term data analysis. BMJ open. 2019;9(8):e030650.

26. Bromfield SG, Ngameni CA, Colantonio LD, Bowling CB, Shimbo D, Reynolds K, et al. Blood Pressure, Antihypertensive Polypharmacy, Frailty, and Risk for Serious Fall Injuries Among Older Treated Adults With Hypertension. Hypertension. 2017;70(2):259-266.

27. Margolis KL, Buchner DM, LaMonte MJ, Zhang Y, Di C, Rillamas-Sun E, et al. Hypertension Treatment and Control and Risk of Falls in Older Women. J Am Geriatr Soc. 2019;67(4):726-733.

28. Yang Y, Hu X, Zhang Q, Zou R. Diabetes mellitus and risk of falls in older adults: a systematic review and meta-analysis. Age Ageing. 2016;45(6):761-767.

29. Neila Calvo S, Nan Nan D, García Ibarbia C, Olmos Martínez JM, González Macías J. Hernández Hernández JL. La realidad de la osteoporosis en el paciente hospitalizado en Medicina Interna. Revista de Osteoporosis y Metabolismo Mineral. 2013:5:141-5.

30. Cerocchi I, Ghera S, Gasbarra E, Scialdoni A, Tarantino U. Fragility fractures: the clinical pathway. Aging Clin Exp Res. 2013;25 Suppl 1:S43-5.

31. Milat F, Ebeling PR. Osteoporosis treatment: a missed opportunity. Med J Aust. 2016;205(4):185-90.

32. Tang BM, Eslick GD, Nowson C, Smith C, Bensoussan A. Use of calcium or calcium in combination with vitamin $\mathrm{D}$ supplementation to prevent fractures and bone loss in people aged 50 years and older: a meta-analysis. Lancet. 2007;370(9588):657-66.

33. Flais J, Coiffier G, Le Noach J, Albert JD, Faccin M, Perdriger A, Thomazeau H, Guggenbuhl P. Low prevalence of osteoporosis treatment in patients with recurrent major osteoporotic fracture. Arch Osteoporos. 2017;12(1):24.

34. Kim SC, Kim MS, Sanfélix-Gimeno G, Song HJ, Liu J, Hurtado I, Peiró S, et al. Use of osteoporosis medications after hospitalization for hip fracture: a cross-national study. Am J Med. 2015;128(5):519-26.e1.

35. Tinetti ME, Kumar C. The patient who falls: "It's always a trade-off". JAMA. 2010;303(3):258-66.

36. Rubenstein LZ. Falls in older people: epidemiology, risk factors and strategies for prevention. Age Ageing. 2006;35 Suppl 2:ii37-ii41.

37. Rubenstein LZ, Josephson KR. Falls and their prevention in elderly people: what does the evidence show? Med Clin North Am. 2006;90(5):807-24.

38. Belfrage B, Koldestam A, Sjöberg C, Wallerstedt SM. Number of drugs in the medication list as an indicator of prescribing quality: a validation study of 
polypharmacy indicators in older hip fracture patients. Eur J Clin Pharmacol. 2015;71(3):363-8.

39. Lönnbro J, Wallerstedt SM. Clinical relevance of the STOPP/START criteria in hip fracture patients. Eur J Clin Pharmacol. 2017;73(4):499-505.

40. Gosch M, Wörtz M, Nicholas JA, Doshi HK, Kammerlander C, Lechleitner M. Inappropriate prescribing as a predictor for long-term mortality after hip fracture. Gerontology. 2014;60(2):114-22.

41. Machado-Alba JE, Gaviria-Mendoza A, Machado-Duque ME, Chica L. Deprescribing: a new goal focused on the patient. Expert Opin Drug Saf. 2017;16(2):111-112.

42. Machado-Duque ME, Castaño-Montoya JP, Medina-Morales DA, Castro-Rodríguez A, González-Montoya A, Machado-Alba JE. Association between the use of benzodiazepines and opioids with the risk of falls and hip fractures in older adults. Int Psychogeriatr. 2018;30(7):941-946.

43. Machado-Duque ME, Castaño-Montoya JP, Medina-Morales DA, Castro-Rodríguez A, González-Montoya A, Machado-Alba JE. Drugs With Anticholinergic Potential and Risk of Falls With Hip Fracture in the Elderly Patients: A Case-Control Study. J Geriatr Psychiatry Neurol. 2018;31(2):63-69 